CERN-'TII-5230/88

\title{
LOOKING FOR THE EFFECTS OF DYNAMICAL QUARKS
}

\author{
Apoorva Palel \\ Theory Division, CERN, Geneva, Switzerland
}

Talk presented at the International Symposium "Lattice 88 "

Fermilab, USA, September 22-25, 1988

\section{ABSTRACT}

I present our recent. Monte Carlo results demonstrating the effects of dynamical quarks on the hadron spectrum.

CFR.N-TH-5230/88

November 1988 
For those of you not interested in technical details, the main outcome of what 1 am going to describe is shown in Fig. 3. The rest of the paper describes the methods we used, the checks we performed and the comparisons we made on our way to reach that stage.

We incorporated the effect of dynamical quarks into our simulation of the functional integral using the hybrid Monte Carlo algorithm ${ }^{1}$. This algorithm corresponds to evolving the system through a fictitious (computer) time. One starts with an initial configuration and Langevin noise as the initial momentum. The system is then evolved in discrete time steps using reversible and phase space area preserving equations of molecular dynamics for a certain trajectory length. For a step size $\epsilon$, this classical evolution introduces an $O\left(\epsilon^{2}\right)$ in the Hamiltonian. In order to make the algorithm exact, one gets rid of this error by a global accept/reject of the final configuration with the standard Metropolis criterion. This procedure is iterated for many such trajectories in order to span out the phase space. The efficiency of the algorithm is dictated by the dependence of $\epsilon$ on the system volume $V$, quark mass $m$ and the gauge coupling $\beta \equiv 6 / g^{2}$, when the rate of acceptance is held fixed. The volume dependence of the algorithm has been studied ${ }^{2}$, and asymptotically $\epsilon$ is found to depend on the volume as $V^{-1 / 4}$, i.c., the computer time scales as $V^{5 / 4}$.

The algorithm spends most of its time inverting the fermion matrix $M$ in the form $\chi=\left(M^{\dagger} M\right)^{-1} \phi$. Therefore the dependence of the algorithm on the fermion sector, especially its slow-down at small quark masses is an important question to address. We have explored several aspects of this problem:

1. The rate of convergence of the iterative procedure for matrix inversion (we used the conjugate gradi- ent method) typically depends on the small eigenvalues of the matrix being inverted. Therefore for a fixed convergence criterion, such as

$$
\frac{\left\|\left(M^{\dagger} M\right) \chi-\phi\right\|^{2}}{\|\chi\|^{2}}<R,
$$

we expect the number of iterations and the computer time for each inversion to grow as $1 / \mathrm{m}$. We however discovered that this was not enough, the reason being that the relevant accuracy is not the one defined by $R$, but is the accuracy to which $\delta I I$ - the change in the Hamiltonian upon which the accept/reject step depends - is evaluated. $\delta H$ is calculated as the difference of two large numbers of $O(V)$ and for a sensible acceptance one must have $\delta I I=O(1)$. We found that to compute $\delta I I$, say to less than a percent accuracy, it was necessary to decrease $R$ with a reduction in $m$.

2. The size of $\delta H$ and fluctuations in it grow with decreasing $m$. Our empirical observation is that in order to maintain a constant acceptance, it is necessary to make $\epsilon$ smaller at smaller quark masses.

3. There is critical slowing down as $m \rightarrow 0$ (and/or $\beta \rightarrow \infty$ ) in terms of motion through the phase space. We haven't really tested this, but random walk type arguments suggest that the autocorrelation time and hence the computer time should grow as the square of the correlation length. Our results at small quark masses show that autocorrelation times of $50-100$ trajectories are not unusual.

These observations emphasise that it is crucial to study the quark mass dependence of the hybrid Monte Carlo algorithm, and though it is the best algorithm we have 
in our hands right now, it is still prohibitively slow for the present generation computers.

For Wilson fermions the determinant of the fermion matrix is real $\left(\gamma_{5} M^{\dagger} \gamma_{5}=M\right)$ but not positive definite. So to include the determinant as part of the probability factor, one is forced to work with the matrix $M^{\dagger} M$, i.e., double the number of quark flavours. Our results ${ }^{3}$ have been obtained with two flavours of $r=1$ dynamical Wilson quarks at $\beta=5.3$ on an $8^{4}$ lattice. We used periodic boundary conditions for the gauge field and anti-periodic ones for the quarks. The hybrid Monte Carlo algorithm allows the molecular dynamics parameters governing the classical trajectory to be different from the Monte Carlo ones controlling the Boltzmann distribution. For our leap-frog scheme, choosing the two sets to be equal is close to the optimal scenario, while a shift to $\beta_{m d}=5.29$ gave a slightly better acceptance. The trajectory length was selected so as to obtain the best decorrelation time. The matrix inversions were carried out after initialising $\chi$ with a guess extrapolated from the previous two time steps. The momenta were exponentiated exactly by first diagonalising the matrix. We tried to monitor the algorithmic errors by inspecting the values of $\delta H$.

de Forcrand has raised an objection to all small step size algorithms that they are highly inefficient in tunnelling through the zero modes of the determinant. This is a valid point, but its effect is likely to show up only close to the chiral limit, when zero modes separating sectors of different topological charge (the usual index theorem connection) really start influencing the physics. Even the smallest quark mass we have worked with is too heavy to see the influence of zero modes on tunnelling. In particular, we calculated the winding number of our configurations using the cooling technique and found it to be fluctuating between 0 and 4 .

We have calculated Wilson loops on our lattices at six values of the hopping parameter $\kappa: 0.156,0.158$, $0.160,0.162,0.165$ and 0.167 . Each new run was started from a thermalised configuration at the previous lower value of $\kappa$ (a pure gauge configuration in the case of $\kappa=0.156$ ). The prominent features of the results are:

1. Approximate algorithms that are not corrected for finite step size errors (such as pseudofermion, Langevin and hybrid) appear to have underesti-

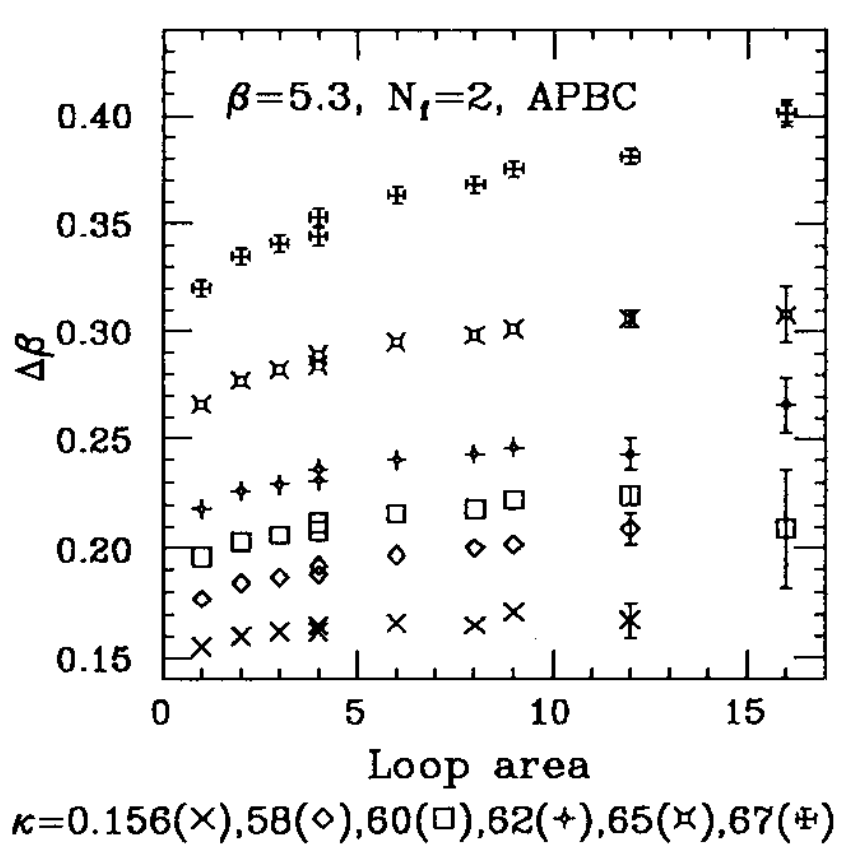

Figure 1: The shift in the gauge coupling required to match the Wilson loop results for full QCD with those of the pure gauge $\mathrm{SU}(3)$ theory vs. the area of the loop.

mated the effects of dynamical quarks. Their results have to be extrapolated to the zero step size limit $^{4}$ to match them onto exact ones and these extrapolations become larger for bigger Wilson loops.

2. Wilson fermions show a stronger departure from the pure gauge theory results than staggered fermions ${ }^{5}$, for the same number of quark flavours and the same lattice quark mass. Though the comparison is crude because the mass renormalisation is not identical for the two fermion schemes, the difference is very obvious. This effect is easily explainable. The staggered fermion flavour symmetry is far from being exact at the strong gauge coupling we have worked with, i.e., the effective number of staggered quark flavours is smaller than its naively assumed value.

3. It is not possible to match the Wilson loop results with dynamical quarks to those of the pure gauge theory with a shifted gauge coupling. Figure 1 shows the shift in the gauge coupling necessary for such a matching plotted against the Wilson loop size at various values of the quark mass. At short distances dynamical quarks essentially renormalise 
Table 1: The statistical parameters

\begin{tabular}{llll}
$\kappa$ & 0.162 & 0.165 & 0.167 \\
\hline Trajectory length & 0.8 & 0.75 & 0.75 \\
Step size & 0.04 & 0.0375 & 0.03 \\
Metropolis acceptance & 0.62 & 0.47 & 0.31 \\
Thermalisation & 150 & 180 & 190 \\
No. of configurations & 30 & 30 & 60 \\
Configuration separation & 50 & 60 & 40 \\
$R_{\text {update }}$ & $10^{-10}$ & $10^{-11}$ & $10^{-12}$ \\
$R_{\text {hadron }}$ & $10^{-12}$ & $10^{-12}$ & $10^{-14}$
\end{tabular}

the gauge coupling. But beyond the $m$-dependent string breaking scale, one expects to see the difference in the asymptotic nature of the two theories. The increase in $\Delta \beta$ with the loop size reflects the change from area law to perimeter law, while a matching would require $\Delta \beta$ to be the same for loops beyond a certain size typified by the confinement scale. The figure shows that though the matching is almost possible for heavy quark masses (corresponding to a large string breaking scale), at smaller quark masses there is clear eviderice against simultaneous matching of all Wilson loops.

For three values of the dynamical quark mass, corresponding to $\kappa_{\text {sea }}=0.162,0.165$ and 0.167 , hadron propagators were calculated on these configurations. The parameters of this calculation are listed in Table 1 (thermalisation and configuration separation are in units of molecular dynamics trajectories). The temporal boundary conditions were taken to be periodic and the matrix inversions were carried out to higher accuracy than in the configuration update part. We used local hadron operators and discovered that it was necessary to triple the lattice size to $8^{3} \times 24$ in order to see asymptotic fall-off of the hadron correlators. We observe that the baryon correlators take a longer time to reach the asymptotic region than the meson ones, in agreement with ref. 6. A typical case is illustrated in Fig. 2, where the ratio of the nucleon propagator on adjacent time-slices is plotted vs. time on lattices doubled and tripled in the time direction.

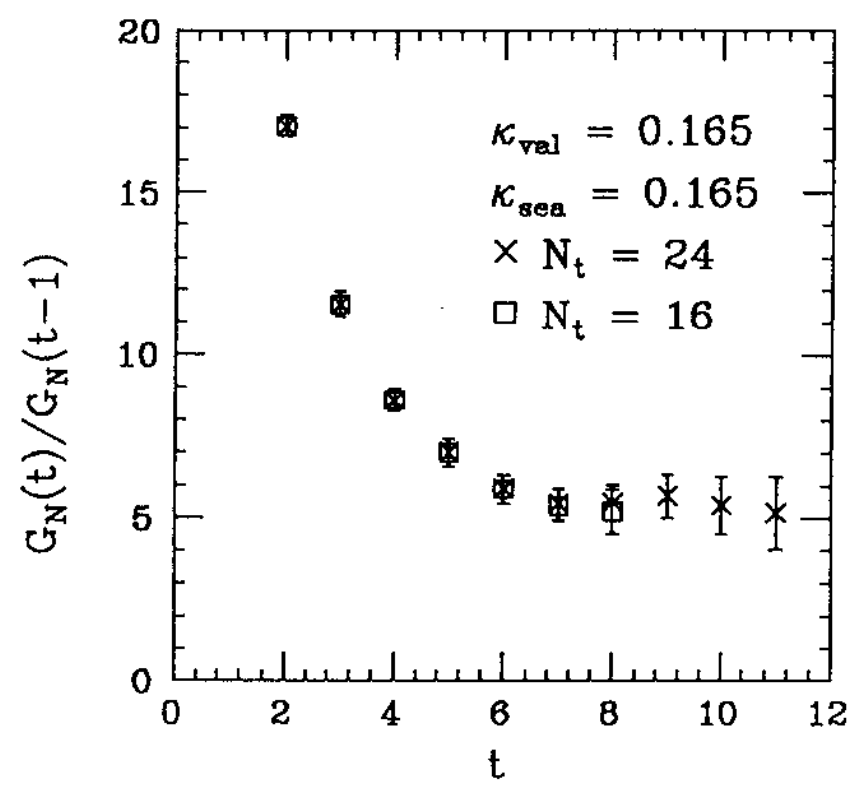

Figure 2: Approach of the nucleon propagator towards its asymptotic behaviour on lattices doubled and tripled in the time direction.

Since the dynamical quark mass and the valence quark mass, characterised by $\kappa_{s e a}$ and $\kappa_{\text {yal }}$ respectively, are independent parameters in the lattice calculation, we extended the hadron spectrum results to the case $\kappa_{\text {sea }} \neq \kappa_{\text {yal }}$ at the lightest $(\kappa=0.167)$ and the heaviest $(\kappa=0.162)$ sea quark masses. The results for $\pi, \rho, N$ and $\Delta$ masses are presented in Table 2. All the results are with stable double exponential fits in the region $3 \leq t \leq 12$ and only the statistical errors are shown. The non-zero momentum contribution to the baryon masses due to anti-periodic spatial boundary conditions has been subtracted off using the lattice dispersion relation :

$$
M^{2}=E^{2}-\sin ^{2} p_{x}-\sin ^{2} p_{y}-\sin ^{2} p_{z} .
$$

Our estimates for the critical hopping parameter and the lattice scale are $\kappa_{c}=0.1686(3)$ and $a^{-1}=1.4(2) \mathrm{GeV}$.

All of our results correspond to fairly heavy quarks, the lowest quark mass roughly being that of the strange quark. Due to lack of any direct experimental information on the hadron masses in this region, it has become popular to compare the lattice results to those of phenomenological models. A simple and convenient parametrisation is that of the quark model with hyper- 
Table 2: The hadron masses

\begin{tabular}{cccccc}
$\kappa_{\text {val }}$ & $\kappa_{\text {sca }}$ & $\pi$ & $\rho$ & $N$ & $\Delta$ \\
\hline 0.167 & 0.162 & $0.89(1)$ & $1.03(1)$ & $1.75(6)$ & $1.80(8)$ \\
0.165 & 0.162 & $0.96(1)$ & $1.08(1)$ & $1.80(6)$ & $1.84(7)$ \\
0.162 & 0.162 & $1.05(1)$ & $1.15(6)$ & $1.87(5)$ & $1.89(6)$ \\
0.165 & 0.165 & $0.79(1)$ & $0.93(1)$ & $1.52(7)$ & $1.61(8)$ \\
0.167 & 0.167 & $0.49(2)$ & $0.68(2)$ & $1.12(7)$ & $1.22(7)$ \\
0.165 & 0.167 & $0.58(1)$ & $0.74(2)$ & $1.19(7)$ & $1.26(7)$ \\
0.162 & 0.167 & $0.71(1)$ & $0.83(1)$ & $1.29(7)$ & $1.34(7)$
\end{tabular}

fine mass splittings ${ }^{7}$

$$
\begin{aligned}
& M_{m}=C_{m}+\sum_{i=1}^{2} m_{i}+\xi_{m} \frac{\vec{S}_{1} \cdot \vec{S}_{2}}{m_{1} m_{2}}, \\
& M_{b}=C_{b}+\sum_{i=1}^{3} m_{i}+\xi_{b} \sum_{i>j} \frac{\vec{S}_{i} \cdot \vec{S}_{j}}{m_{i} m_{j}} .
\end{aligned}
$$

Here $m_{i}, \vec{S}_{i}$ represent constituent mass and spin of the quarks, while the subscripts $m, b$ stand for mesons and baryons respectively. The constants $C$ and $\xi$ are determined by fitting $(3,4)$ to the experimental hadron masses and they depend on the hadron wave-functions, in particular on the reduced mass of each quark pair and the presence of sea quarks. The Goldstone nature of the pseudo-scalar mesons is not correctly incorporated in (3). Yet the formulae do a good job on fitting the masses of hadrons involving $u, d$ and $s$ quarks. (The $\eta^{\prime}$ is an exception because of the triangle anomaly effect.) These quarks cover the region where the hadron wavefunctions are by and large independent of the quark mass : $M_{\pi} / M_{\rho} \in[0.2,0.7]$. For the heaviest quark mass point in this region, the fit parameters produce $M_{\Omega} / M_{\phi}=1.62$ to be compared with the experimental value 1.64. However, it should not be ignored that outside this region the formulae do not work as well (e.g., when the charm quark mass is fit to reproduce the $D$ and $\Lambda_{c}$ masses, the masses of $\tilde{c} c$ mesons are off by $7-10 \%$ ), and this is where our numerical results lie.

Having pondered on this state of ignorance, we can now look at the data for $M_{N} / M_{\rho}$ in Fig. 3. I emphasise that the numbers quoted in this paper are not to be taken quantitatively, since the systematic errors in the

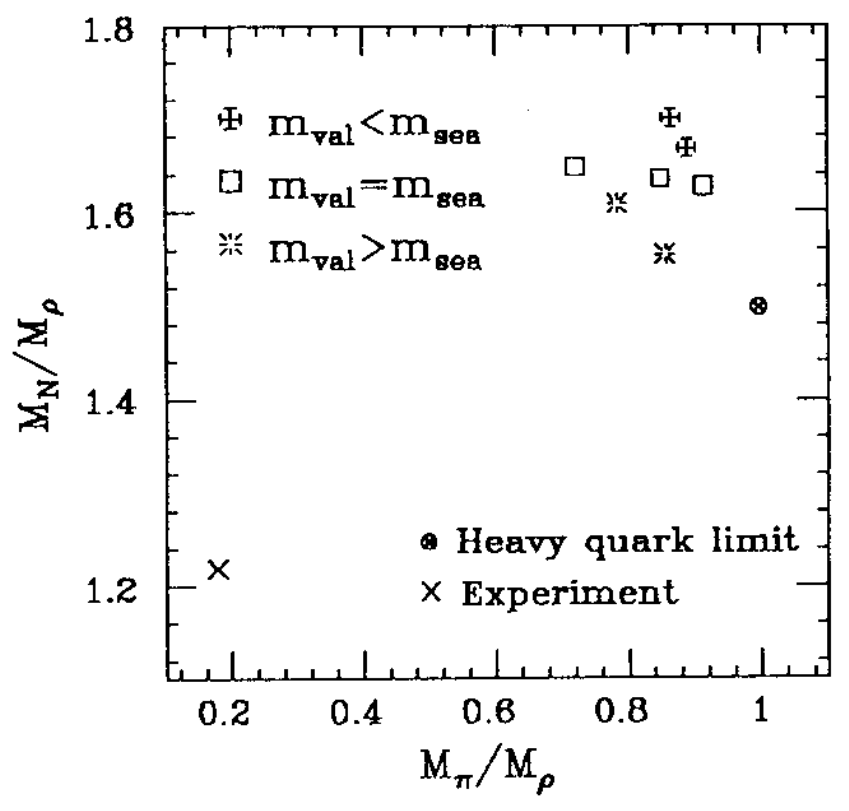

Figure 3: Monte Carlo results depicting the effect of dynamical quarks on the nucleon to rho mass-ratio.

results - such as strong coupling lattice artifacts, finite volume effects, fitting biases, algorithmic uncertainties, inadequate statistics etc. - far outweigh the naive statistical errors at this stage. There is a long way to go before obtaining any predictions for the real world. For simulation of all hadrons the sea quarks have to be light, whether the valence quarks are light (as in the case of $\pi, \rho$ and $N$ ) or heavy (as in the case of charm and bottom quark systems). Nonetheless a qualitative feature of the data that unambiguously stands out even in the heavy quark mass region is the relative order of the results. The systematic errors listed above are not likely to affect this order, since it is based on similar kind of data having undergone an identical analysis procedure. Particularly the three points at $M_{\pi} / M_{\rho} \approx 0.85$ show that at a fixed value of $M_{\kappa} / M_{\rho}$, lowering the the sea quark mass decreases $M_{N} / M_{\rho}$. This is a highly welcome feature in view of the fact that numerical studies of the quenched theory $\left(m_{\text {sec }}=\infty\right)$ have consistently produced too large a value for $M_{N} / M_{\rho}$. It should be possible to qualitatively confirm this effect in strong coupling expansions where statistical and finite volume errors as well as fitting uncertainties are absent. I am pursuing this investigation further to shed more light on the role of sea quarks inside hadrons, particularly the nucleon. 


\section{ACKNOWLEDGEMENTS}

Most of the work described here was accomplished on the new Cray X-MP/48 at CERN during its teething period. 1 thank the CERN DD-division for their assistance with the computational facilities. I am grateful to my collaborators for their help and advice.

\section{REFERENCES}

1. S. Duane, A. Kennedy, B. Pendleton and D. Roweth, Phys. Lett. B195 (1987) 216

2. R. Gupta, G. Kilcup and S. Sharpe, Phys. Rev. D38 (1988) 1278;

M. Creutz, Phys. Rev. D38 (1988) 1228
3. R. Gupta, A. Patel, C. Baillie, G. Guralnik, G. Kilcup and $S$. Sharpe, in preparation;

A. Patel, R. Gupta, G. Kilcup and S. Sharpe, in preparation

4. M. Fukugita, Y. Oyanagi and A. Ukawa, Phys. Rev. D36 (1987) 824

5. S. Gottlieb, W. Liu, D. Toussaint, R. Renken and R. Sugar, Phys. Rev. D35 (1987) 3972

6. Y. Iwasaki and T. Yoshie, University of Tsukuba preprint, UTHEP-182 (1988)

7. S. Ono, Phys. Rev. D17 (1978) 888;

A. De Rujula, H. Georgi and S. Glashow, Phys. Rev. D12 (1975) 147 